
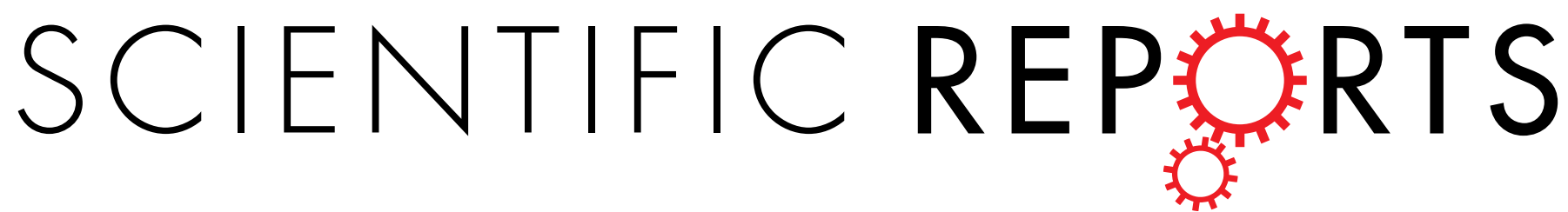

\title{
OPEN Room Temperature Quantum Spin Hall Insulator in Ethynyl-Derivative Functionalized Stanene Films
}

Received: 29 May 2015

Accepted: 30 November 2015

Published: 05 January 2016

\author{
Run-wu Zhang ${ }^{1}$, Chang-wen Zhang ${ }^{1}$, Wei-xiao $\mathrm{Ji}^{1}$, Sheng-shi Li ${ }^{1}{ }^{2}$, Shi-shen Yan ${ }^{2}$, Shu-jun $\mathrm{Hu}^{2}$, \\ Ping $\mathrm{Li}^{1}$, Pei-ji Wang ${ }^{1}$ \& Feng $\mathrm{Li}^{1}$
}

Quantum spin Hall (OSH) insulators feature edge states that topologically protected from backscattering. However, the major obstacles to application for QSH effect are the lack of suitable QSH insulators with a large bulk gap. Based on first-principles calculations, we predict a class of large-gap $\mathrm{QSH}$ insulators in ethynyl-derivative functionalized stanene ( $\mathrm{SnC}_{2} \mathrm{X} ; \mathrm{X}=\mathrm{H}, \mathrm{F}, \mathrm{Cl}, \mathrm{Br}$, I), allowing for viable applications at room temperature. Noticeably, the $\mathrm{SnC}_{2} \mathrm{Cl}, \mathrm{SnC}_{2} \mathrm{Br}$, and $\mathrm{SnC}_{2} \mathrm{I}$ are $\mathrm{QSH}$ insulators with a bulk gap of $\sim 0.2 \mathrm{eV}$, while the $\mathrm{SnC}_{2} \mathrm{H}$ and $\mathrm{SnC}_{2} \mathrm{~F}$ can be transformed into $\mathrm{OSH}$ insulator under the tensile strains. A single pair of topologically protected helical edge states is established for the edge of these systems with the Dirac point locating at the bulk gap, and their QSH states are confirmed with topological invariant $Z_{2}=1$. The films on $B N$ substrate also maintain a nontrivial large-gap QSH effect, which harbors a Dirac cone lying within the band gap. These findings may shed new light in future design and fabrication of large-gap OSH insulators based on two-dimensional honeycomb lattices in spintronics.

Topological insulators (TIs) ${ }^{1-4}$ have generated intensive research and engineering activities in recent years, because they give an alternative and robust platform for obtaining relativistic and spin-polarized Fermions in the condensed matter system. One of the most interesting phenomena in this realm is the quantum spin Hall (QSH) effect in two-dimensional (2D) structures ${ }^{1,2}$, characterized by an insulating bulk and gapless edge states at its boundaries due to time-reversal symmetry (TRS), thus providing enticing concepts for novel quantum devices with low energy dissipation ${ }^{3,4}$. The prototypical concept of QSH effect is first proposed by Kane and Mele in graphene ${ }^{5,6}$, in which the spin-orbit coupling (SOC) opens a band gap at the Dirac point. However, the associated gap due to rather weak SOC is too small $\left(\sim 10^{-3} \mathrm{meV}\right)$, which makes the QSH state in graphene only appear at an unrealistically low temperature. To date, quantized conductance through QSH state has only been experimentally observed in $\mathrm{HgTe} / \mathrm{CdTe}^{7,8}$ and InAs/GaSb ${ }^{9,10}$ quantum wells at the ultralow temperature, which greatly obstructs further experimental studies and possible applications in spintronics.

Generally, a good QSH insulator should be easily synthesized and have a large bulk band gap to realize the spin transport at high temperatures. Controlling the chemical bonding at atomic levels to induce the band inversion by orbital selection $^{3,4}$ is an efficient way to realize QSH effect ${ }^{8,11-15} .2 \mathrm{D}$ materials are advantageous in this respect as their bonding character is relatively easy to modify in post synthesis processes, for example, by surface adsorption. Recently, group-IV honeycomb lattices such as functionalized germanene and stanene ${ }^{16,17}$ have been reported, with a bulk-gap as large as $0.2-0.3 \mathrm{eV}$, sufficiently large for practical applications at room temperature. Besides, the strong SOC effect can also be sufficed by group-V heavy elements such as bismuth, which drives nontrivial QSH states $^{18,19}$. On the other hand, group III-V materials GaAs and GaBi have also reported to be large-gap QSH insulators ${ }^{20,21}$. Unfortunately, the experiments ${ }^{22}$ reveal that plasma fluorination and hydrogenation generally increase defects and lattice disorder even under short plasma exposures. Thus, the achievement of these systems with high quality is rather challengeable, demonstrating the detection of QSH effect is difficult in experiments. More recently, an approach to design a large-gap QSH state on a semiconductor surface by a substrate orbital filtering process is also proposed ${ }^{23,24}$. These large-gap QSH insulators are essential for realizing many exotic phenomena and for fabricating new quantum devices that can operate at room temperature.

${ }^{1}$ School of Physics and Technology, University of Jinan, Jinan, Shandong, 250022, People's Republic of China. ${ }^{2}$ School of Physics, State Key laboratory of Crystal Materials, Shandong University, Jinan, Shandong, 250100, People's Republic of China. Correspondence and requests for materials should be addressed to C.-W.Z. (email: zhchwsd@163. com) 

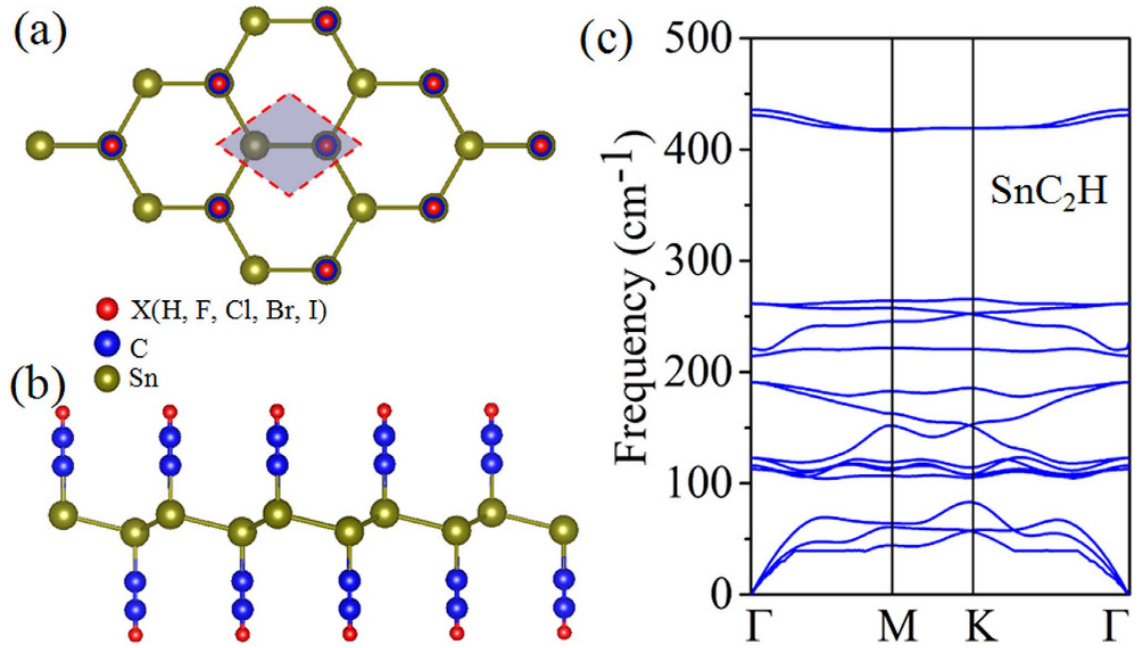

Figure 1. (a) Side and (b) top views of the atomic structures of $\mathrm{SnC}_{2} \mathrm{X}(\mathrm{X}=\mathrm{H}, \mathrm{F}, \mathrm{Cl}, \mathrm{Br}, \mathrm{I})$. Red, blue, and green balls denote X, C, and Sn atoms, respectively. Shadow area in (a) present the unit cell. Phonon band dispersions of (c) denote $\mathrm{SnC}_{2} \mathrm{H}$ film.

\begin{tabular}{|l|c|c|c|c|c|c|}
\hline System & $a(\AA)$ & $d_{1}(\AA)$ & $d_{2}(\AA)$ & $h(\AA)$ & $E_{g}(\mathrm{meV})$ & Phase \\
\hline Stanene & 4.674 & 2.830 & - & 0.852 & 93 & TI \\
\hline $\mathrm{SnC}_{2} \mathrm{H}$ & 4.756 & 2.851 & 2.108 & 0.766 & 28 & $\mathrm{NI}$ \\
\hline $\mathrm{SnC}_{2} \mathrm{~F}$ & 4.734 & 2.843 & 2.102 & 0.782 & 25 & $\mathrm{NI}$ \\
\hline $\mathrm{SnC}_{2} \mathrm{Cl}$ & 4.871 & 2.891 & 2.109 & 0.718 & 192 & $\mathrm{TI}$ \\
\hline $\mathrm{SnC}_{2} \mathrm{Br}$ & 4.781 & 2.863 & 2.109 & 0.725 & 202 & $\mathrm{TI}$ \\
\hline $\mathrm{SnC}_{2} \mathrm{I}$ & 4.811 & 2.874 & 2.112 & 0.709 & 234 & $\mathrm{TI}$ \\
\hline
\end{tabular}

Table 1. The lattice constant $a$, Sn-Sn bond length $d_{1}$, Sn-X bond length $d_{2}$, buckling parameters $h$, bulk band gap with SOC $E_{g}$, and phase of the structures investigated in our study. The phase indicates whether the material is a normal insulator (NI) or TI based on the parity calculations.

Recently, the small molecule functionalization has been the focus to enhance the geometric stability and nontrivial band gap of new 2D films. For instance, Methyl $\left(\mathrm{CH}_{3}\right)$, another organic molecule, has also been suggested as a promising tool to stabilize $2 \mathrm{D}$ systems, such as methyl-functionalized germanane $\left(\mathrm{GeCH}_{3}\right)^{25}$ and bismuth $\left(\mathrm{BiCH}_{3}\right)^{26}$, to realize large gap QSH insulators. Experimentally, $\mathrm{GeCH}_{3}$ film has been synthesized in recent work ${ }^{27}$. This raises an interesting question: can the methyl be applied to stabilize group IV and V films, and whether their band gap can be enhanced significantly in QSH phase? Motivated by recent works on stanene by zhang et al. ${ }^{17}$, in our previous works ${ }^{28}$, we have found that the organic molecule ethynyl $\left(\mathrm{C}_{2} \mathrm{H}\right)$ can be applied to stabilize stanene by decoration on its surface, and its band gap in TI states reaches up to $0.3 \mathrm{eV}$. However, the QSH phase is not intrinsic, but driven by the external strain, thus is unfavorable for practical applications in spintronics. Here, we extend these into ethynyl-derivative functionalized films $\left(\mathrm{SnC}_{2} \mathrm{X} ; \mathrm{X}=\mathrm{F}, \mathrm{Cl}, \mathrm{Br}, \mathrm{I}\right)$, making the experimental observation facile. The $\mathrm{SnC}_{2} \mathrm{Cl}, \mathrm{SnC}_{2} \mathrm{Br}$, and $\mathrm{SnC}_{2} \mathrm{I}$ are all QSH insulators with a bulk gap of $\sim 0.2 \mathrm{eV}$, while the $\mathrm{SnC}_{2} \mathrm{H}$ and $\mathrm{SnC}_{2} \mathrm{~F}$ can be transformed into QSH insulator under the tensile strains. The topological characteristic of these films is attributed to $s-p_{x y}$ band inversion related to the honeycomb symmetry, while the effect of SOC is only to open up a large gap. We also propose high-dielectric $\mathrm{BN}$ as an ideal substrate for the experimental realization of these films, maintaining its nontrivial topology. These results have potential applications in low-power quantum electronics and may enable topological quantum computing based on Majorana fermions ${ }^{29}$.

\section{Results and Discussion}

We have performed first-principles calculations of electronic structures and band topology of $2 \mathrm{D}$ hexagonal lattices functionalized with ethynyl-derivatives. We first discuss in detail the results of $\mathrm{SnC}_{2} \mathrm{H}$ and $\mathrm{SnC}_{2} \mathrm{Br}$ as representative examples. Figure 1(a) shows the geometric structure of $2 \mathrm{D} \mathrm{SnC}_{2} \mathrm{X}$, which prefers a buckled configuration, with $\mathrm{C}_{2} \mathrm{X}$ bonding on both sides of stanene in an alternating way (Fig. 1(b)), in analogy to hydrogenated germanene and stanene ${ }^{16,17}$. In comparison to the pristine stanene, the lattice constant of $\mathrm{SnC}_{2} \mathrm{X}$ increase slightly by $0.19 \sim 0.06 \AA$, along with the buckling height between $S n$ planes decreasing by about $0.14 \sim 0.07 \AA$, the details as listed in Table 1 . The buckling plays a crucial role in the engineering of the band structures in this structure. To confirm the structural stability, we calculate the phonon spectrum along the highly symmetric directions, as shown in Fig. 1(c). One can see that all branches of the phonon dispersion curves have positive frequencies and no imaginary phonon modes are found, confirming the stability of $\mathrm{SnC}_{2} \mathrm{H}$ film. 

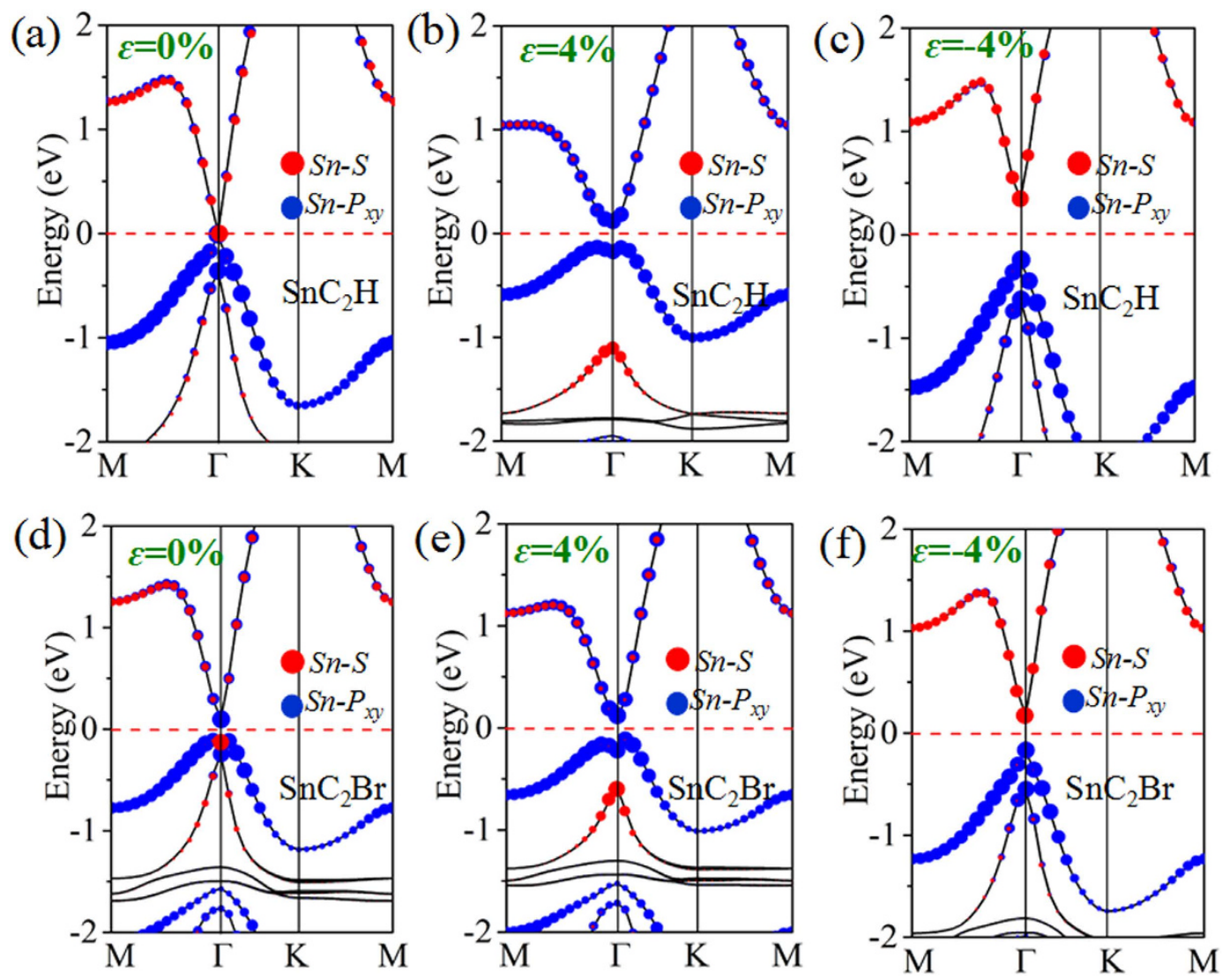

Figure 2. Orbital-resolved band structures with $\mathrm{SOC}$ of $\mathrm{SnC}_{2} \mathrm{H}(\mathrm{a}-\mathrm{c})$ and $\mathrm{SnC}_{2} \mathrm{Br}(\mathrm{d}-\mathrm{f})$ under biaxial strain $\varepsilon=0 \%, 4 \%$ and $-4 \%$, respectively. The red dots represent the contributions from the $s$ atomic orbital of $\mathrm{Sn}$ atom and the blue dots represent contributions from the $p_{\mathrm{x}}$ and $p_{\mathrm{y}}$ atomic orbitals of Sn atom.

\begin{tabular}{|l|c|c|c|c|c|}
\hline $\boldsymbol{\Gamma}_{\boldsymbol{i}}$ & Parity of $\boldsymbol{\xi}_{2 \mathrm{n}}$ of occupied bands & $\boldsymbol{\delta}_{\boldsymbol{i}}$ & $\boldsymbol{\Gamma}_{\boldsymbol{i}}$ & Parity of $\boldsymbol{\xi}_{2 \mathrm{n}}$ of occupied bands & $\boldsymbol{\delta}_{\boldsymbol{i}}$ \\
\hline$(0.0,0.0)$ & +-+-+-+++---+ & + & $(0.0,0.0)$ & ----+-+-----+++---+ & - \\
\hline$(0.5,0.0)$ & +-+-+--+++--+ & + & $(0.5,0.0)$ & ----+-+-----+++- & - \\
\hline$(0.0,0.5)$ & +-+-+--++++-+ & + & $(0.0,0.5)$ & ----+-+-----+++---+ & - \\
\hline$(0.5,0.5)$ & -+-+-++---++- & - & $(0.5,0.5)$ & -----+-+----+---++- & + \\
\hline$\varepsilon \geq 2.0 \% \mathrm{SnC}_{2} \mathrm{H}$ & $\mathbf{Z}_{2}$ topological invariant & $\boldsymbol{\nu}=\mathbf{1}$ & $\varepsilon \geq-2.0 \% \mathrm{SnC}_{2} \mathrm{Br}$ & $\mathrm{Z}_{2}$ topological invariant & $\boldsymbol{\nu}=\mathbf{1}$ \\
\hline
\end{tabular}

Table 2. Parities of occupied spin-degenerate bands at the TRIM Points for $\mathrm{SnC}_{2} \mathrm{H}$ and $\mathrm{SnC}_{2} \mathrm{Br}$. Here, we display the parities of 13 occupied spin-degenerate bands for $\mathrm{SnC}_{2} \mathrm{H}(\varepsilon \geq 2.0 \%)$ and the parities of 19 occupied spin-degenerate bands for $\mathrm{SnC}_{2} \mathrm{Br}(\varepsilon \geq-2.0 \%)$. Positive and negative signs denote even and odd parities, respectively. The sign in parentheses is the product of the parity eigenvalues of the occupied spin-degenerate bands.

To get a preliminary insight into the topological properties, we present the calculated band structures of $\mathrm{SnC}_{2} \mathrm{H}$ including SOC. At equilibrium state in Fig. 2(a), which shows a semiconductor nature with a direct band gap located at $\Gamma$ point. By projecting the bands onto different atomic orbitals, we find that the valence band maximum $(\mathrm{VBM})$ at $\Gamma$ point near the Fermi level is mainly composed of $p_{\mathrm{x}, \mathrm{y}}$ orbitals from $\mathrm{Sn}$ atoms with the features of binding states, whereas the conduction band minimum (CBM) is the anti-binding $s$ state. Thus, no inverted band order is observed, suggesting that it is a trivial TI phase. However, if applying the tensile strain $(\varepsilon=4 \%)$, the $s-p$ band inversion appears, with an indirect-gap of $0.22 \mathrm{eV}$ opened under SOC effect, as illustrated in Fig. 2(b). To demonstrate the 2D TI phase, we calculate the $\mathrm{Z}_{2}$ invariants $v$, as listed in Table 2. Obviously, the products of the parity eigenvalues at these three symmetry points: $\Gamma(0.0,0.0), M_{1}(0.5,0.0)$ and $M_{2}(0.0,0.5)$ are both 1 , while at the $\mathrm{M}_{3}(0.5,0.5)$ shows -1 , which give $\mathrm{Z}_{2}=1$. Therefore, it directly confirms the existence of QSH insulator state.

The buckled configuration generally sustain a larger mechanical strain than planner one, thus we explore the variations of electronic structure with respect to the mechanical strain ${ }^{30-34}$. Here, we employ an external strain on $\mathrm{SnC}_{2} \mathrm{H}$ maintaining the crystal symmetry by changing its lattices as $\varepsilon=\left(\mathrm{a}-\mathrm{a}_{0}\right) / \mathrm{a}_{0}$, where $\mathrm{a}\left(\mathrm{a}_{0}\right)$ is the strained (equilibrium) lattice constants. In what follows, we systematically investigate the effects of both uniaxial and biaxial strains on topological properties of $\mathrm{SnC}_{2} \mathrm{H}$. Figure 3(a) presents the variation of nontrivial QSH gap (direct, $E_{\Gamma}$ ) at 

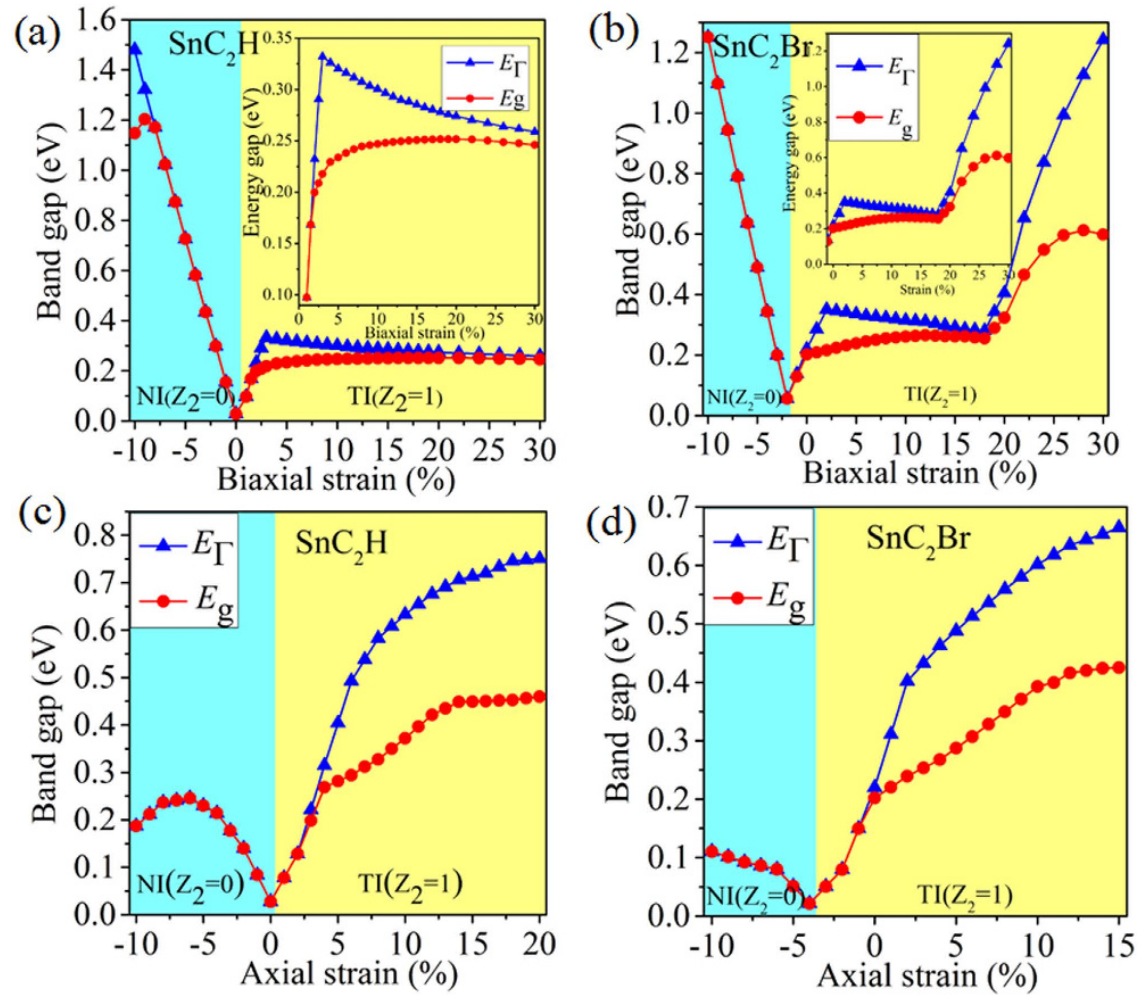

Figure 3. The calculated band gaps at $\Gamma$ point $\left(E_{\Gamma}\right)$ and the global band gap $\left(E_{\mathrm{g}}\right)$ of $\mathrm{SnC}_{2} \mathrm{H}$ and $\mathrm{SnC} \mathrm{Br}_{2} \mathrm{Bith}$ SOC as a function of biaxail and uniaxial strains. Notably, the insets in panel show the trend of band gaps of TI phase as a function of external biaxial strain.

$\Gamma$ point and bulk gap (indirect, $E_{\mathrm{g}}$ ) as a function of the biaxial strain. One can see that, both the direct and indirect band gaps in TI phase decrease steadily with respect to strain, especially $E_{\mathrm{g}}$ and $E_{\Gamma}$ being equal to strain beyond $30 \%$. Remarkably, for $\varepsilon=3 \%$, we find the largest gap of $\sim 0.33 \mathrm{eV}$ opened at $\Gamma$ point, along with a global indirect band gap of $\sim 0.22 \mathrm{eV}$, which is significantly greater than $k_{B} T(\sim 26 \mathrm{meV})$. While the biaxial strain is compressed to $\varepsilon=-4 \%$, we find that no inverted band order is observed, suggesting that it is a trivial QSH insulator (Fig. 2(c)). On the other hand, we explore the effect of uniaxial strain on the electronic properties, as displayed in Fig. 3(c). Interestingly, we obtain a larger SOC-induced bulk gap as large as $\sim 0.46 \mathrm{eV}(\varepsilon=18 \%)$, which is almost twice the one of biaxial strain. Further analysis of the parity eigenvalue gives $Z_{2}=1$, which suggests that the uniaxial strain is also efficient way to tune QSH state in these films.

Now, we wish to point out that the ethynyl functionalization in stanene is not the only way to achieve the large-gap QSH state, the same results can be obtained by decorating the surface with otherwise ethynyl-derivatives, such as $-\mathrm{C}_{2} \mathrm{~F},-\mathrm{C}_{2} \mathrm{Cl},-\mathrm{C}_{2} \mathrm{Br}$, and $-\mathrm{C}_{2} \mathrm{I}$. We thus perform calculations for $\mathrm{SnC}_{2} \mathrm{X}(\mathrm{X}=\mathrm{F}, \mathrm{Cl}, \mathrm{Br}$, I) films to check their topological properties. Table 1 summarizes their lattice constants, $\mathrm{Sn}$-Sn bond lengths, and nontrivial gaps at their equilibrium states. Similar to the case of $\mathrm{SnC}_{2} \mathrm{H}$, for $\mathrm{SnC}_{2} \mathrm{~F}$, it is a trivial QSH phase at the equilibrium state, but can be driven to QSH phase at the biaxial tensile strain $\varepsilon>2 \%$. However, for the other $\mathrm{SnC}_{2} \mathrm{X}(\mathrm{X}=\mathrm{Cl}, \mathrm{Br}, \mathrm{I})$ films, they are TI phases at the equilibrium state with $\mathrm{Z}_{2}=1$, as listed in Table 2. The global QSH gaps of $\mathrm{SnC}_{2} \mathrm{Cl}$, $\mathrm{SnC}_{2} \mathrm{Br}$, and $\mathrm{SnC}_{2} \mathrm{I}$ are $0.19,0.20$, and $0.23 \mathrm{eV}$, respectively, (see Table 1 and supplemental information in Fig. S1), which are sufficiently large for practical application at high temperature.

The calculated band structures of $\mathrm{SnC}_{2} \mathrm{Br}$ film are presented in Fig. 2(d-f) as a typical model. Obviously, the $s-p_{x y}$ band inversion are still exist, in analogy to the strain-induced QSH phase in $\mathrm{SnC}_{2} \mathrm{H}$. Similar results for $\mathrm{SnC}_{2} \mathrm{~F}$, $\mathrm{SnC}_{2} \mathrm{Cl}$, and $\mathrm{SnC}_{2} \mathrm{I}$ films are also observed, as illustrated in Fig. S1. From Fig. 3(b), we also find that the biaxial-strain range of QSH phase is rather large (from - 3\% to 30\%). Interestingly, there are two peaks in the region of the topology phase, in which one peak is $0.34 \mathrm{eV}$ for $E_{\mathrm{g}}$ under $3 \%$ strain, and another higher peak is $0.61 \mathrm{eV}$ at $28 \%$. Such robust topology makes it easier for experimental realization in spintronics. For uniaxial strain (Fig. 3(c-d)), we consider the effects of uniaxial strain along $\mathrm{x}$ direction for $\mathrm{SnC}_{2} \mathrm{H}$ and $\mathrm{SnC}_{2} \mathrm{Br}$. In addition, the detailed evolution of band structure for $\mathrm{SnC}_{2} \mathrm{H}$ under the uniaxial strain is added into Fig. S2. Band structure calculations indicate that it is still in QSH phase, with a larger bulk gap of $\sim 0.42 \mathrm{eV}(\varepsilon=16 \%)$. Also, as can be seen in Fig. S3, the systematically investigate the effects of both uniaxial and biaxial strains on topological properties of $\mathrm{SnC}_{2} \mathrm{~F}, \mathrm{SnC}_{2} \mathrm{Cl}$ and $\mathrm{SnC}_{2} \mathrm{I}$, respectively, which are sufficiently tunable ranges of strain for practical applications at high temperature.

The QSH phase should support topologically protected conducting edge states that are helical with the spin-momentum locked by TRS. To demonstrate these edge states explicitly, we take $\mathrm{SnC}_{2} \mathrm{H}$ and $\mathrm{SnC}_{2} \mathrm{Br}$ with the external strain $\varepsilon=2 \%$ and $\varepsilon=0 \%$, respectively, to introduce edges on stanene by forming the zigzag-type nanoribbons, as shown in Fig. 4(a). The edge Sn atoms are passivated by hydrogen atoms to eliminate the dangling 


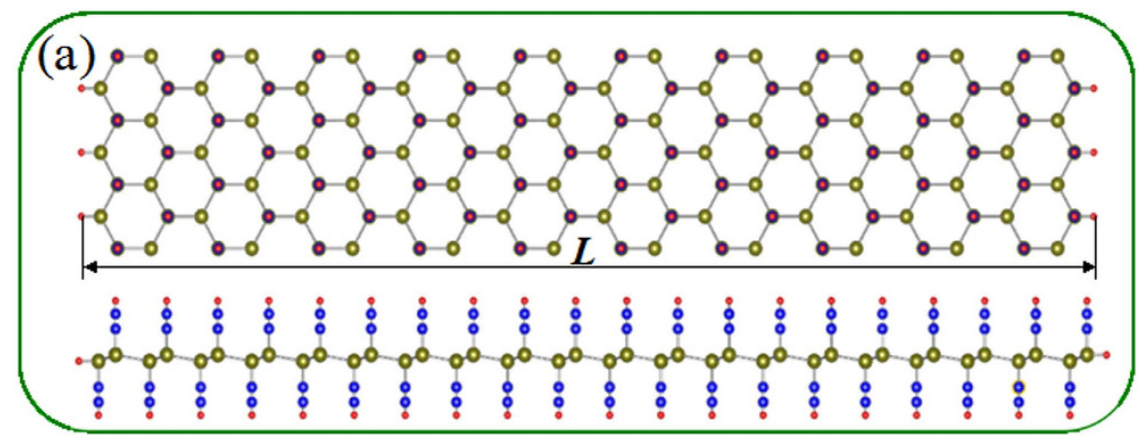

(b)

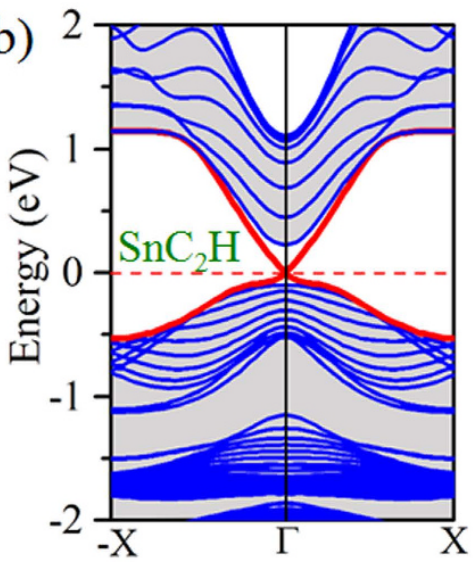

(c)

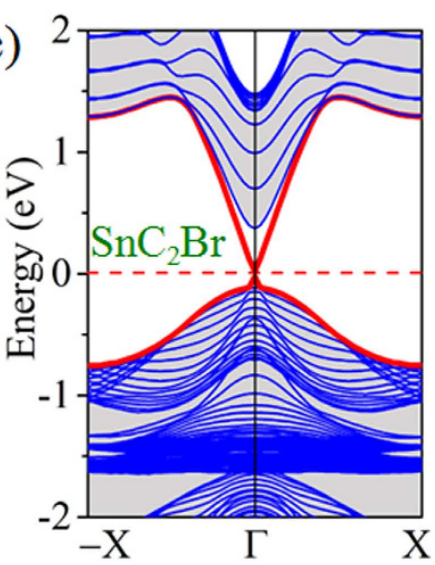

Figure 4. (a) Schematic structure of the zigzag-type nanoribbon of $\mathrm{SnC}_{2} \mathrm{H}$ and $\mathrm{SnC}_{2} \mathrm{Br}$ films. (b) and (c) indicate the band structures of zigzag-type edge states in QSH phase. The helical edge states are indicated by the red lines.

bonds. The width of the nanoribbon is large enough to avoid interactions between the edge states of the two sides. Figure 4(b,c) show the zigzag-type edge states of $\mathrm{SnC}_{2} \mathrm{X}(\mathrm{X}=\mathrm{H}, \mathrm{Br})$ films, where a Dirac point at the $\Gamma$ point is located inside the band gap with a high velocity of $\sim 1.0 \times 10^{5} \mathrm{~m} / \mathrm{s}$ and $\sim 2.0 \times 10^{5} \mathrm{~m} / \mathrm{s}$, comparable to that of $5.5 \times 10^{5} \mathrm{~m} / \mathrm{s}$ in $\mathrm{HgTe} / \mathrm{CdTe}$ quantum well ${ }^{35}$, both of which are larger than that of $3.0 \times 10^{4} \mathrm{~m} / \mathrm{s}$ in InAs/GaSb quantum well ${ }^{36}$. Helical edge states are very useful for electronics and spintronics owning to their robustness against scattering. The similar results for the remaining edge states of $\mathrm{SnC}_{2} \mathrm{X}(\mathrm{X}=\mathrm{F}, \mathrm{Cl}, \mathrm{I})$ are also displayed in Fig. S4. All these band structures maintain topologically protected conducting edge states that are helical, thus providing enticing concepts for novel quantum electronic devices with low energy dissipation.

It is known that GGA method usually underestimates the band gap, we thus carried out test computations based on the hybrid functional HSE0 $6^{37,38}$ to assess the robustness of our results within the PBE. The calculated band structures of $\mathrm{SnC}_{2} \mathrm{X}(\mathrm{X}=\mathrm{Cl}, \mathrm{Br}, \mathrm{I})$ films under the critical strain are presented in Fig. S3 in supplementary information. One can see that the band gap of these films are indeed enhanced. However, the $s-p_{x, y}$ band order is not changed and in agreement with those obtained from PBE, indicating the PBE results are qualitatively reasonable and reliable.

To better understand the physical origin of QSH states, we next do an orbital analysis around the Fermi level for $\mathrm{SnC}_{2} \mathrm{X}$ films. Figure 5 presents the band evolution at $\Gamma$ point, in which the energy levels near the Fermi level are mainly composed of $S n-5 s$ and $S n-5 p_{x, y}$ orbitals. Thus, the chemical bonding between $S n-S n$ atoms makes them split into the bonding and antibonding states, i.e., $s^{ \pm}$and $p_{\mathrm{x}, \mathrm{y}}{ }^{ \pm}$, where the superscripts + and - represent the parities of corresponding states, respectively. At zero strain in Fig. 5(a), the bands near the Fermi level are contributed by the $p_{\mathrm{x}, \mathrm{y}}{ }^{+}$and $s^{-}$, with the $s^{-}$being above the ${p_{\mathrm{x}, \mathrm{y}}}^{+}$, indicating a normal band order, as shown in $\mathrm{SnC}_{2} \mathrm{H}$. When considering the tensile strain, as seen in Fig. 5(b), the enlarged lattice constant weakens the interaction between $\mathrm{Sn}$ atoms, decreasing the splitting between the bonding and antibonding states, which lowers $s^{-}$level and raises $p_{\mathrm{x}, \mathrm{y}}{ }^{+}$ level accordingly. Thus, depending on the strength of interatomic coupling, the band gap of $\mathrm{SnC}_{2} \mathrm{H}$ can be continuously reduced, with the band order being reversed at critical point, making it a topological insulator with $\mathrm{Z}_{2}=1$. Taken together, the level crossing leads to a parity exchange between occupied and unoccupied bands, inducing a TI phase transition. While for $\mathrm{SnC}_{2} \mathrm{Br}$, the lattice constant at equilibrium state is equal to strain-induced $\mathrm{SnC}_{2} \mathrm{H}$ $(\varepsilon=4 \%)$, thus it is a QSH insulator. As a result, the $s-p$ band inversion ${ }^{39}$ is a strong indication of the existence of topologically nontrivial phases. Herein, we must point out that, although the $s-p_{x, y}$ band inversion is caused mainly by the strength of interatomic coupling, the SOC is still indispensable because it makes the $p_{\mathrm{x}, \mathrm{y}}{ }^{+}$orbital split into

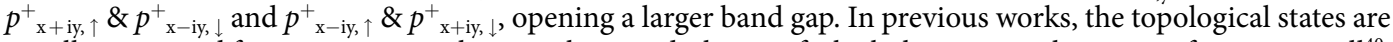
usually originated from $p_{\mathrm{z}}$ system such as graphene and silicene of which the nontrivial gaps are often very small ${ }^{40}$. but in our cases, the topological states with larger gaps are obtained due to the contribution of $p_{\mathrm{x}, \mathrm{y}}$ orbitals on $\mathrm{Sn}$ 
(a)

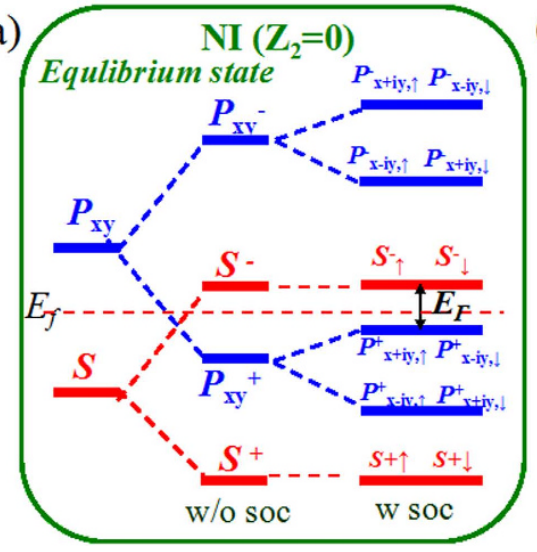

(b)

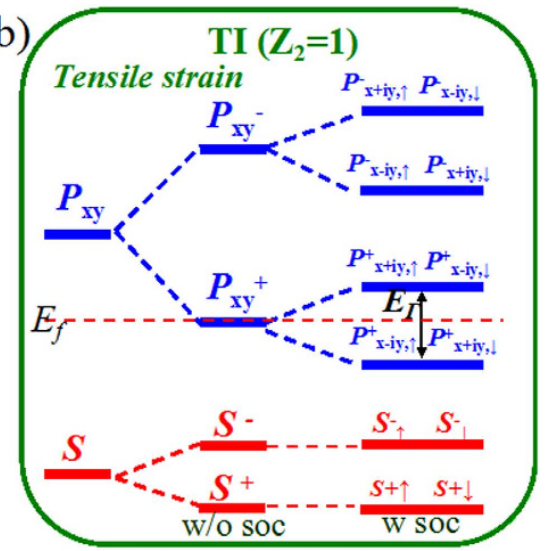

Figure 5. The evolution of atomic $s$ and $p_{\mathrm{x}, \mathrm{y}}$ orbitals into the band edges at $\Gamma$ point of $\operatorname{SnC}_{2} \mathrm{X}(\mathrm{X}=\mathrm{H}, \mathrm{F})$ at (a) equilibrium state, and (b) tensile strain $(\varepsilon=\mathbf{2} \%)$. The horizontal red dashed lines in $(\mathbf{a}, \mathbf{b})$ indicate the Fermi level.

(a)

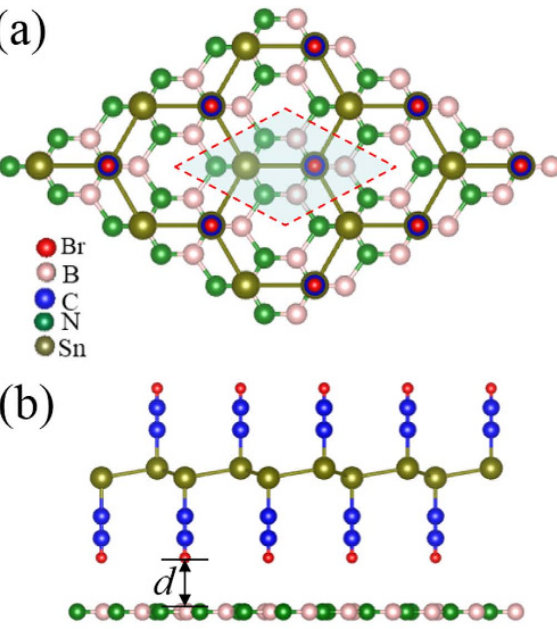

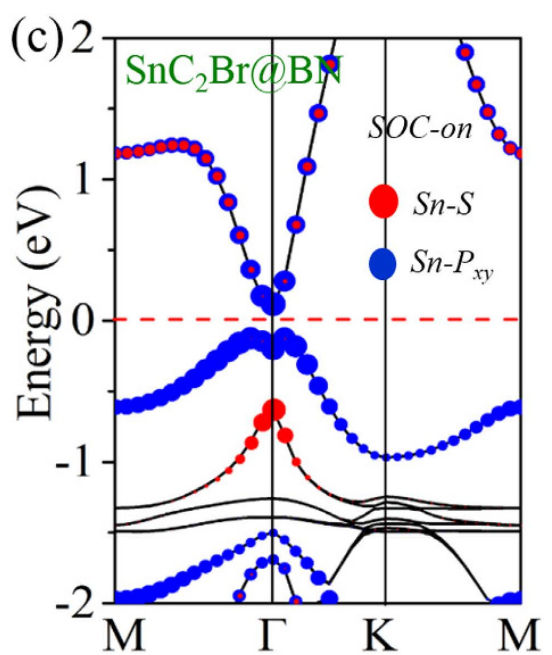

Figure 6. (a) Top and (b) side views of the schematic illustration of the epitaxial growth $\mathrm{SnC}_{2} \mathrm{Br}$ on $h$ - $\mathrm{BN}$ $(2 \times 2)$ substrate. $(\mathbf{c})$ is orbital-resolved band structure with SOC for $\mathrm{SnC}_{2} \mathrm{Br} @ \mathrm{BN}$.

atoms, where the large SOC is from the on-site SOC, instead of the next-nearest-neighbor SOC in the $p_{\mathrm{z}}$ cases $^{40}$. These results emphasize the importance of orbital selection for topological materials.

On the experimental side, choosing the suitable substrate material is a key factor in device application, since a free-standing film must eventually be deposited or grown on a substrate. It is known that the QSH features in pristine graphene and germanene ${ }^{12-17}$ are easily destroyed by the substrate. In contrast, although the TI phase of $\mathrm{SnC}_{2} \mathrm{X}$ films are obtained in free-standing structure, their nontrivial QSH would be robust when they are on the substrate, because their band inversion occurs at $\Gamma$ point rather than at $K$ point, as well as the full saturation of $\mathrm{Sn}-p_{\mathrm{z}}$ orbitals ensures a weak interaction with the substrate. Here, we select $h$-BN as a possible substrate to form $\mathrm{SnC}_{2} \mathrm{Br} @ \mathrm{BN}$ heterostructure (HTS), where the lattice mismatch is only about $1.19 \%\left(\mathrm{SnC}_{2} \mathrm{Br}\right)$ in comparison to $h$ - $\mathrm{BN}(2 \times 2)$ sheet, showing that they are feasible to grown on the $h$-BN substrate. To correctly describe the effect of van der Waals (vdW) interaction, we employ a dispersion-corrected DFT method (optB88-vdW) ${ }^{41,42}$, which has been demonstrated to reliably describe 2D HTSs. Thus, the optimized distance $(d)$ between adjacent layers is 3.278 A. The calculated binding energy is found to be $-71 \mathrm{meV}$, suggesting a typical vdW HTS. In Fig. 6(c), we present the corresponding band structure for $\mathrm{SnC}_{2} \mathrm{Br}$ film. Bader analysis ${ }^{43}$ indicates no charge transfer between adjacent layers, thus the states near the Fermi level are mainly determined by $\mathrm{SnC}_{2} \mathrm{Br}$ film. In comparison to the free-standing film, little difference is observed between them. As a result, it is indeed a robust QSH insulator with band inversion not being affected by the $h$-BN substrate. Similar electronic properties of $\mathrm{SnC}_{2} \mathrm{X} @ \mathrm{BN}(\mathrm{X}=\mathrm{H}, \mathrm{F}$, $\mathrm{Cl}, \mathrm{I}) \mathrm{HTS}$ are obtained, as shown in Fig. S6.

To conclude, on the basis of first-principles calculations, we predict a class of new QSH insulator of $\mathrm{SnC}_{2} \mathrm{X}$ films with sizable bulk gaps, making the experimental observation facile. Noticeably, the $\mathrm{SnC}_{2} \mathrm{Cl}, \mathrm{SnC}_{2} \mathrm{Br}$, and $\mathrm{SnC}_{2} \mathrm{I}$ are all QSH insulators with global gaps of $\sim 0.2 \mathrm{eV}$, while the $\mathrm{SnC}_{2} \mathrm{H}$ and $\mathrm{SnC}_{2} \mathrm{~F}$ can be transformed into QSH insulator under the tensile strains. This large-gap opening is mainly attributed to the result of the strong SOC related to the 
$p_{\mathrm{x}}$ and $p_{\mathrm{y}}$ orbitals of $\mathrm{Sn}$ atoms at $\Gamma$ point, significantly different from that of the $p_{\mathrm{z}}$ orbital as in pristine group IV ones. A single pair of topologically protected helical edge states are established for the edge of these systems with the Dirac point locating at the bulk band gap, and their QSH states are also confirmed with $\mathrm{Z}_{2}=1$. Also, these films on h-BN substrate are observed to support a nontrivial large-gap QSH, which harbors a Dirac cone lying within the band gap. These findings may shed new light in future design and fabrication of large-gap QSH insulators based on $2 \mathrm{D}$ honeycomb lattice in spintronics.

\section{Methods}

To study the structural and electronic properties of $\mathrm{SnC}_{2} \mathrm{X}$ films, we employed state-of-the-art ab initio simulations, based on density functional theory (DFT) as implemented in VASP ${ }^{44,45}$. We used the generalized gradient approximation for the exchange and correlation potential, as proposed by Perdew-Burk-Ernzerhof (PBE) ${ }^{46}$, the projector augmented wave potential $(\mathrm{PAW})^{47}$ to treat the ion-electron interactions. The energy cutoff of the plane waves was set to $500 \mathrm{eV}$ with the energy precision of $10^{-6} \mathrm{eV}$. The Brillouin zone was sampled by using a $21 \times 21 \times 1$ Gamma-centered Monkhorst-Pack grid. The vacuum space was set to 20 Å to minimize artificial interactions between neighboring slabs. SOC was included by a second vibrational procedure on a fully self-consistent basis. With the optimized structures, the more sophisticated HSE06 hybrid functional ${ }^{37,38}$ was used to check the corresponding results of the systems. The phonon spectra were calculated using a supercell approach within the PHONON code ${ }^{48}$. To determine the $\mathrm{Z}_{2}$ index of the inversion symmetric $2 \mathrm{D}$ materials, the invariants $v$ can be derived from the parities of wave function at the four TRIM points $\mathrm{K}_{\mathrm{i}}$, namely one $\Gamma$ point and three equivalent $\mathrm{M}$ points in the Brillouin zone. The topological invariant $\nu$ was determined based on the Fu-Kane's formula ${ }^{49}$ :

$$
\delta\left(K_{i}\right)=\prod_{m=1}^{N} \xi_{2 m}^{i},(-1)^{\nu}=\prod_{i=1}^{4} \delta\left(K_{i}\right)=\delta(\Gamma) \delta(M)^{3}
$$

where $\delta$ is the product of parity eigenvalues at the TRIM points, $\xi= \pm 1$ are the parity eigenvalues and $N$ is the number of the occupied bands. According to the $\mathrm{Z}_{2}$ classification, $\nu=1$ characterizes a QSH insulator, whereas $\nu=0$ represents a trivial band topology.

\section{References}

1. Yan, B. \& Zhang, S. Topological Materials. Rep. Prog. Phys. 75, 096501 (2012).

2. Moore, J. E. Majorana’s Wires. Nat. Nanotechnol 8, 194 (2013).

3. Song, Z. et al. Quantum spin Hall insulators and quantum valley Hall insulators of $\mathrm{BiX} / \mathrm{SbX}(\mathrm{X}=\mathrm{H}, \mathrm{F}, \mathrm{Cl}$ and $\mathrm{Br})$ monolayers with a record bulk band gap. NPG Asia Mater. 6, e147 (2014).

4. Qi, X. \& Zhang, S. Topological insulators and superconductors. Rev. Mod. Phys. 83, 1057 (2011).

5. Kane, C. L. \& Mele, E. J. Quantum Spin Hall Effect in Graphene. Phys. Rev. Lett. 95, 226801 (2005).

6. Kane, C. L. \& Mele, E. J. Z Topological Order and the Quantum Spin Hall Effect. Phys. Rev. Lett. 95, 146802 (2005).

7. Bernevig, B. A., Hughes, T. L. \& Zhang, S. Quantum Spin Hall Effect and Topological Phase Transition in HgTe Quantum Wells. Science. 314, 1757 (2006).

8. König, M. et al. Quantum Spin Hall Insulator State in HgTe Quantum Wells. Science 318, 766 (2007).

9. Du, L., Knez, I., Sullivan, G. \& Du, R. Robust Helical Edge Transport in Gated InAs/GaSb Bilayers. Phys. Rev. Lett. 114, 096802 (2015).

10. Liu, C. et al. Quantum Spin Hall Effect in Inverted Type-II Semiconductors. Phys. Rev. Lett. 100, 236601 (2008).

11. Qi, X. \& Zhang, S. The quantum spin Hall effect and topological insulators. Rhys. Today 63, 33 (2010).

12. Fu, L., Kane, C. L. \& Mele, E. J. Topological Insulators in Three Dimensions. Phys. Rev. Lett. 98, 106803 (2007).

13. Zhang, H. et al. Topological insulators in $\mathrm{Bi}_{2} \mathrm{Se}_{3}, \mathrm{Bi}_{2} \mathrm{Te}_{3}$ and $\mathrm{Sb}_{2} \mathrm{Te}_{3}$ with a single Dirac cone on the surface. Nat. Phys. 5, 438 (2009).

14. Wu, S., Shan, G. \& Yan, B. Prediction of Near-Room-Temperature Quantum Anomalous Hall Effect on Honeycomb Materials. Phys. Rev. Lett. 113, 256401 (2014).

15. Tang, P. et al. Stable two-dimensional dumbbell stanene: A quantum spin Hall insulator. Phys. Rev. B 90, 121408 (2014).

16. Si, C. et al. Functionalized Germanene as a Prototype of Large-gap Two-dimensional Topological Insulators. Phys. Rev. B 89, 115429 (2014).

17. Xu, Y. et al. Large-Gap Quantum Spin Hall Insulators in Tin Monolayers. Phys. Rev. Lett. 111, 136804 (2013).

18. Liu, C. et al. Topological Phase Transitions in Half-hydrogenated Bi Honeycomb Monolayers. Phys. Rev. B 90, 085431 (2014).

19. Jin, K. H. \& Jhi, S. H. Quantum Anomalous Hall and Quantum Spin-Hall Phases in Flattened Bi and Sb Bilayers. Sci. Rep. 5, 8426 (2015).

20. Li, L., Zhang, X., Chen, X. \& Zhao, M. Giant Topological Nontrivial Band Gaps in Chloridized Gallium Bismuthide. Nano Lett. 15, 1296 (2015).

21. Zhao, M., Chen, X., Li, L. \& Zhang, X. Driving a GaAs Film to a Large-gap Topological Insulator by Tensile Strain. Sci. Rep. 5, 8441(2015)

22. Wu, J. et al. Controlled Chlorine Plasma Reaction for Noninvasive Graphene Doping. J. Am. Chem. Soc. 133, 19668 (2011).

23. Zhou, M. et al. Formation of quantum spin Hall state on Si surface and energy gap scaling with strength of spin orbit coupling. Sci. Rep. 4, 7102 (2014).

24. Zhou, M. et al. Epitaxial growth of large-gap quantum spin Hall insulator on semiconductor surface. PNAS 111, 14378 (2014).

25. Ma, Y. et al. Strain-induced quantum spin Hall Effect in methyl-substituted germanane $\mathrm{GeCH}_{3}$. Sci. Rep. 4, 7297 (2014).

26. Ma, Y. et al. Robust Two-Dimensional Topological Insulators in Methyl-Functionalized Bismuth, Antimony, and Lead Bilayer Films. Nano Lett. 15(2), 1083 (2015).

27. Jiang, S. et al. Improving the stability and optical properties of germanane via one-step covalent methyl-termination. Nat. Commun. 5, 3389 (2014).

28. Zhang, R. et al. Ethynyl-functionalized stanene film: a promising candidate as large gap quantum spin Hall insulator. New J. Phys. 17, 083036 (2015).

29. Tsai, W. et al. Gated Silicene as a Tunable Source of Nearly 100\% Spin-Polarized Electrons. Nat. Commun. 4, 1500 (2013).

30. Zhu, Z., Cheng, Y. \& Schwingenschlögl, U. Topological phase transition in layered GaS and GaSe. Phys. Rev. Lett. 108, 266805 (2012).

31. Li, Y. \& Wu, C. High-dimensional topological insulators with quaternionic analytic Landau levels. Phys. Rev. Lett. 110, 216802 (2013).

32. Murakami, S., Nagaosa. N. \& Zhang, S. Dissipationless quantum spin current at room temperature. Science 301, 1348 (2003).

33. Zhao, L., Liu, J. W., Tang, P. Z. \& Duan, W. H. Design of Strain-engineered Quantum Tunneling Devices for Topological Surface States. Appl. Phys. Lett. 100, 131602 (2012).

34. Ma, Y., Dai, Y., Yu, L., Niu, C. \& Huang, B. Engineering a Topological Phase Transition $\beta$-InSe via Strain. New J. Phys. 15, 073008 (2013). 
35. Konig M. et al. Quantum spin Hall insulator state in HgTe quantum wells. Science 318, 1148047 (2007)

36. Du, L., Knez, I., Sullivan, G. \& Du, R. R. Robust Helical Edge Transport in Gated InAs/GaSb Bilayers. Phys. Rev. Lett. 114, 096802 (2015).

37. Heyd, J., Scuseria, G. E. \& Ernzerhof, M. J. Hybrid functionals based on a screened Coulomb potential. J. Chem. Phys. 118, 8207 (2003).

38. Heyd, J., Scuseria, G. E. \& Ernzerhof, M. J. Erratum: Hybrid functionals based on a screened Coulomb potential. J. Chem. Phys. 124, 219906 (2006).

39. Zimmermann, H., Keller, R. C., Meisen, P. \& Seelmann-Eggebert, M. Growth of Sn Thin Films on CdTe (111). Surf. Sci. 904, 377 (1997).

40. Qiao, Z. et al. Quantum anomalous Hall effect in graphene proximity coupled to an antiferromagnetic insulator. Phys. Rev. Lett. 112, 116404 (2014).

41. Klimeš, J., Bowler, D. R. \& Michaelides, A. Chemical accuracy for the van der Waals density functional. J. Phys. Condens. Matter. 22, 022201 (2010).

42. Klimeš, J., Bowler, D. R. \& Michaelides, A. Van der Waals density functionals applied to solids. Phys. Rev. B 83, 195131 (2011).

43. Bader, R. F. W. A quantum theory of molecular structure and its applications. Chem. Rev. 91, 893 (1991).

44. Kresse, G. \& Furthmüller, J. Efficient iterative schemes for ab initio total-energy calculations using a plane-wave basis set. Phys. Rev. B 54, 11169 (1996).

45. Kresse, G. \& Furthmüller, J. Efficiency of ab-initio total energy calculations for metals and semiconductors using a plane-wave basis set. Comput. Mater. Sci. 6, 15 (1996).

46. Perdew, J. P., Burke, K. \& Ernzerhof, M. Generalized Gradient Approximation Made Simple. Phys. Rev. Lett. 77, 3865 (1996).

47. Blöchl, P. E. Projector augmented-wave method. Phys. Rev. B 50, 17953 (1994).

48. Zimmermann, H., Keller, R. C., Meisen, P. \& Seelmann-Eggebert, M. Growth of Sn thin films on CdTe (111). Surf. Sci. 904, 377 (1997).

49. Fu, L. \& Kane, C. L. Topological Insulators with Inversion Symmetry. Phys. Rev. B 76, 045302 (2007).

\section{Acknowledgements}

We would like to thank Dr. Hongbin Zhang for useful discussions. This work was supported by the National Natural Science Foundation of China (Grant No. 11274143, 11434006, 61172028, and 11304121), and Research Fund for the Doctoral Program of University of Jinan (Grant no. XBS1433).

\section{Author Contributions}

R.Z. and C.Z. conceived the study and wrote the manuscript. R.Z. and S.L. performed the first-principles calculations. S.Y. gave us further discussion. W.J. and S.H. calculated the phonon spectrum. P.L. prepared Figures 1-3, P.W. and F.L. prepared Figures 4-6. All authors read and approved the final manuscript.

\section{Additional Information}

Supplementary information accompanies this paper at http://www.nature.com/srep

Competing financial interests: The authors declare no competing financial interests.

How to cite this article: Zhang, R.-w. et al. Room Temperature Quantum Spin Hall Insulator in EthynylDerivative Functionalized Stanene Films. Sci. Rep. 6, 18879; doi: 10.1038/srep18879 (2016).

(c) (1) This work is licensed under a Creative Commons Attribution 4.0 International License. The images or other third party material in this article are included in the article's Creative Commons license, unless indicated otherwise in the credit line; if the material is not included under the Creative Commons license, users will need to obtain permission from the license holder to reproduce the material. To view a copy of this license, visit http://creativecommons.org/licenses/by/4.0/ 